DESY 96-241

December 1996

HD-THEP-96-50

\title{
Magnetic Screening in the High Temperature Phase of the Standard Model
}

\author{
W. Buchmüller \\ Deutsches Elektronen-Synchrotron DESY, 22603 Hamburg, Germany \\ O. Philipsen \\ Institut für Theoretische Physik, Universität Heidelberg, 69120 Heidelberg, Germany
}

\begin{abstract}
Non-perturbative effects in the high-temperature phase of the electroweak theory are characterized by a magnetic screening length. Its size influences the range of validity of perturbation theory, and it also determines the critical Higgs boson mass where the first-order phase transition changes to a crossover. We propose a gauge-invariant definition of the magnetic screening length and discuss its role in several gauge-dependent and gauge-invariant correlation functions.
\end{abstract}


The electroweak phase transition [四] is of great cosmological significance because baryon number and lepton number violating processes come into thermal equilibrium as the temperature approaches the critical temperature of the transition [2]. In recent years the thermodynamics of the phase transition has been studied in detail by means of perturbation theory and numerical lattice simulations. As a first step towards the treatment of the full standard model, one usually studies the pure SU(2) Higgs model neglecting the effects of fermions and the mixing between photon and neutral vector boson, which can be included perturbatively. We now know that the transition is firstorder for Higgs boson masses below $70 \mathrm{GeV}$, and that around $\sim 80 \mathrm{GeV}$ the first-order transition changes to a crossover円.

The electroweak transition is influenced by non-perturbative effects whose size is governed by a 'magnetic screening length', the inverse of a 'magnetic mass'. In perturbation theory a magnetic mass appears as a cutoff which regularizes infrared divergencies [4]. The size of this cutoff is closely related to the confinement scale of the effective threedimensional theory which describes the high-temperature limit of the SU(2) Higgs model [5.

In a previous paper [6] we have determined a gauge-independent 'magnetic mass' from the exponential fall-off of the gauge boson propagator by means of gap equations. Contrary to perturbation theory, a mass gap was found in the symmetric phase. A direct consequence was the prediction that the first-order phase transition should turn into a crossover at a critical Higgs mass below $100 \mathrm{GeV}$. Recently, such a crossover behaviour has indeed been observed in numerical lattice simulations for Higgs masses larger than about $80 \mathrm{GeV}$ [7, 8]. However, contrary to the expectation in [6], the 'magnetic mass' was not seen in the correlation functions of gauge-invariant operators which have been studied in detail in numerical simulations [9, 10, 11, 12, 13] and which yield much larger masses. A 'magnetic mass' was only seen in the numerical study of the gauge boson propagator in a fixed (Landau) gauge [12]. In the following we shall make some conjectures which may help to resolve this puzzle and to clarify the physical picture of the symmetric phase.

\section{Magnetic mass and crossover}

Consider the SU(2) Higgs model in three dimensions which is defined by the action

$$
S=\int d^{3} x \operatorname{Tr}\left(\frac{1}{2} W_{\mu \nu} W_{\mu \nu}+\left(D_{\mu} \Phi\right)^{\dagger} D_{\mu} \Phi+\mu^{2} \Phi^{\dagger} \Phi+2 \lambda\left(\Phi^{\dagger} \Phi\right)^{2}\right),
$$

\footnotetext{
${ }^{1}$ For recent reviews, see [3].
} 
with

$$
\Phi=\frac{1}{2}(\sigma+i \vec{\pi} \cdot \vec{\tau}), \quad D_{\mu} \Phi=\left(\partial_{\mu}-i g W_{\mu}\right) \Phi, \quad W_{\mu}=\frac{1}{2} \vec{\tau} \cdot \vec{W}_{\mu} .
$$

Here $\vec{W}_{\mu}$ is the vector field, $\sigma$ is the Higgs field, $\vec{\pi}$ is the Goldstone field and $\vec{\tau}$ is the triplet of Pauli matrices. The gauge coupling $g$ and the scalar coupling $\lambda$ have mass dimension $1 / 2$ and 1 , respectively. For perturbative calculations gauge fixing and ghost terms have to be added. The parameters of the three-dimensional Higgs model have been related to the parameters of the four-dimensional Higgs model at finite temperature by means of dimensional reduction [5]. In particular, variation of temperature corresponds to variation of the mass parameter $\mu^{2}$.

We are interested in the propagators $G_{\sigma}$ and $G_{W}$ of Higgs field and vector field, respectively, which at large separation $|x-y|$ fall off exponentially,

$$
\begin{aligned}
G_{\sigma}(x-y) & =\langle\sigma(x) \sigma(y)\rangle \sim e^{-M|x-y|}, \\
G_{W}(x-y)_{\mu \nu} & =\left\langle W_{\mu}(x) W_{\nu}(y)\right\rangle \sim e^{-m|x-y|} .
\end{aligned}
$$

For $\mu \gg g^{2}$ one has $M \simeq \mu$, whereas $m$ cannot be computed in perturbation theory. A non-vanishing vector boson mass can be obtained from a coupled set of gap equations for Higgs boson and vector boson masses as follows. One shifts the Higgs field $\sigma$ around its vacuum expectation value $v, \sigma=v+\sigma^{\prime}$, which yields the tree level masses

$$
m_{0}^{2}=\frac{g^{2}}{4} v^{2}, M_{0}^{2}=\mu^{2}+3 \lambda v^{2} .
$$

The masses $m_{0}^{2}$ and $M_{0}^{2}$ are now expressed as

$$
m_{0}^{2}=m^{2}-\delta m^{2}, M_{0}^{2}=M^{2}-\delta M^{2}
$$

where $m$ and $M$ enter the propagators in the loop expansion, and $\delta m^{2}$ and $\delta M^{2}$ are treated perturbatively as counter terms. Together with the mass resummation a vertex resummation is performed. One then obtains a coupled set of gap equations for Higgs boson and vector boson masses,

$$
\begin{gathered}
\delta m^{2}+\Pi_{T}\left(p^{2}=-m^{2}, m, M, \xi\right)=0, \\
\delta M^{2}+\Sigma\left(p^{2}=-M^{2}, m, M, \xi\right)=0,
\end{gathered}
$$

where $\Pi_{T}\left(p^{2}\right)$ is the transverse part of the vacuum polarization tensor. The calculation has been carried out in $R_{\xi}$-gauge. In order to obtain masses $M$ and $m$ which are independent of the gauge parameter $\xi$, it is crucial to perform a vertex resummation in addition to the mass resummation and to evaluate the self-energy terms on the mass shell [14]. 
This yields the screening lengths defined in Eq. (3). 'Magnetic masses' defined at zero momentum are gauge-dependent [15].

Together with a third equation for the vacuum expectation value $v$, determined by the condition $\left\langle\sigma^{\prime}\right\rangle=0$, the gap equations determine Higgs boson and vector boson masses for each set of values $\mu^{2} / g^{4}$ and $\lambda / g^{2}$. For negative $\mu^{2}$ one finds a unique solution, corresponding to the familiar Higgs phase, with masses close to the results of perturbation theory. In the case of small positive $\mu^{2} / g^{4}$ and sufficiently small $\lambda / g^{2}$ there exist two solutions, corresponding to the Higgs phase and the symmetric phase with a small, but finite vector boson mass, respectively. This is the metastability range characteristic for a first-order phase transition. For large positive $\mu^{2} / g^{4}$ only the solution corresponding to the symmetric phase remains. Here the Higgs boson mass is $M \simeq \mu$, and the vector boson mass, which is rather independent of $\mu$ and $\lambda$, is given by

$$
\begin{aligned}
m_{S M} & =C g^{2} \\
C & =\frac{3}{64 \pi}(21 \ln 3-4) \simeq 0.28 .
\end{aligned}
$$

Note that this value is rather close to the magnetic mass obtained from gap equations for the pure gauge theory [16, 17] as well as to the three-dimensional confinement scale estimated in [18]. However, the physical interpretation of the magnetic mass is controversial in the case of the pure gauge theory [17]. Furthermore, the magnetic mass (7) is consistent with the propagator mass obtained in a numerical lattice simulation in Landau gauge [12],

$$
m_{S M}^{(L)}=0.35(1) g^{2} .
$$

The mass gap in the symmetric phase is a direct consequence of the non-abelian gauge interactions. In the abelian Higgs model no vector boson mass is generated in the symmetric phase [19].

The size of the magnetic mass determines the critical Higgs boson mass where the first-order transition changes to a crossover. Such a crossover behaviour was first observed for the four-dimensional finite-temperature Higgs model in numerical lattice simulations for large Higgs masses by Evertz, Jersák and Kanaya, who also discussed in detail the phase diagram [20]. In connection with the average action approach the change to a crossover was discussed in [21]. A rough estimate of the critical Higgs mass, where a crossover behaviour sets in, can be obtained as follows. Consider the one-loop effective potential in unitary gauge $(\vec{\pi}=0)$,

$$
V_{1 l}=\frac{1}{2} \mu^{2} \sigma^{2}+\frac{1}{4} \lambda \sigma^{4}-\frac{1}{16 \pi} g^{3} \sigma^{3},
$$




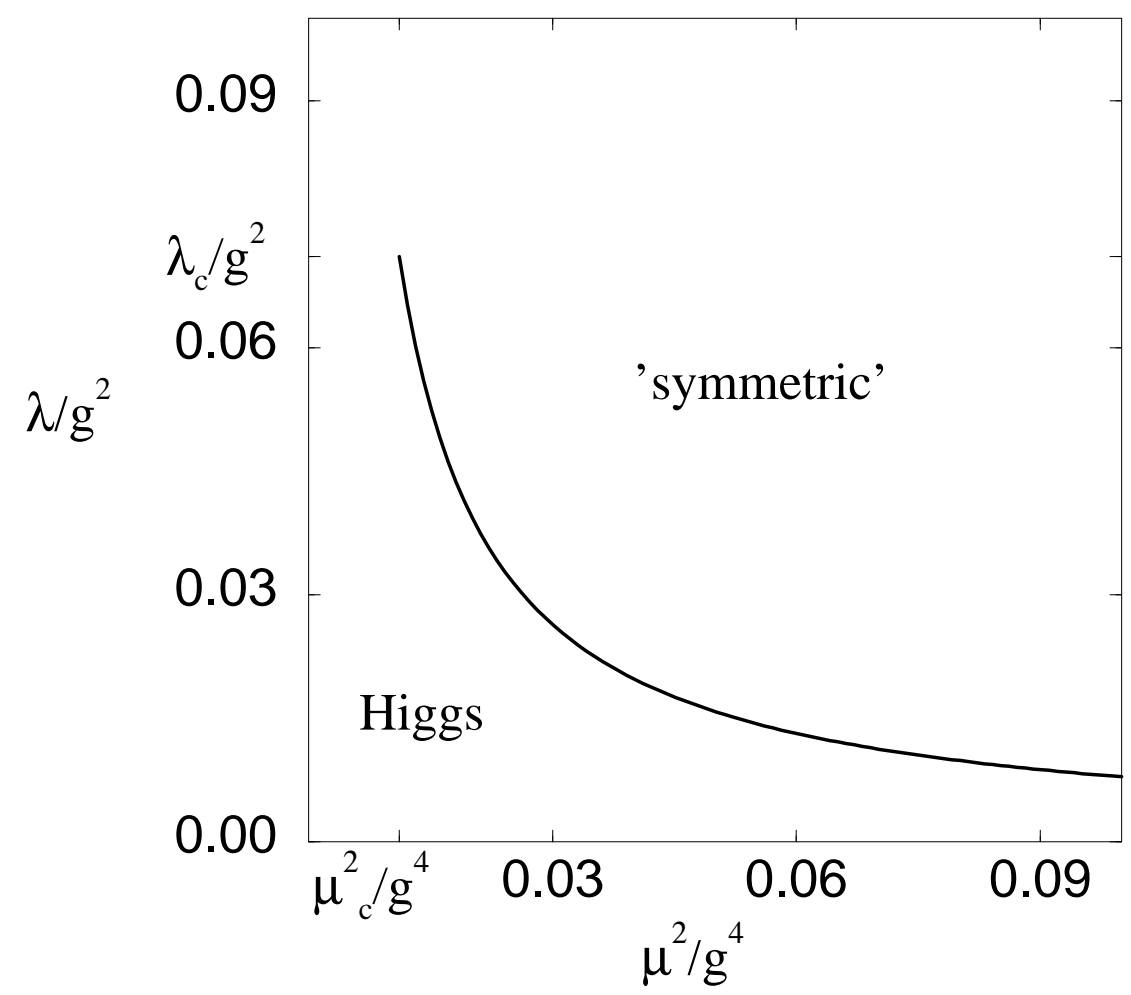

Figure 1: Critical line of first-order phase transitions as given by Eq. (13).

where we have neglected the scalar contributions for simplicity. At the beginning of the metastability range, $\mu^{2}=0$, the Higgs vacuum expectation value is $\sigma_{0}=3 g^{3} /(16 \pi \lambda)$, which corresponds to the vector boson mass

$$
m_{W}\left(\mu^{2}=0\right)=\frac{3}{32 \pi} \frac{g^{4}}{\lambda} .
$$

It is reasonable to expect that the first-order phase transition disappears at a critical scalar coupling where the vector boson mass in the Higgs phase reaches the magnetic mass of the symmetric phase. The condition $m_{W}\left(\mu^{2}=0\right)=m_{S M}$ determines a critical coupling $\lambda_{c}$. The corresponding zero-temperature critical Higgs boson mass is given by

$$
\bar{m}_{H}^{c}=\left(\frac{3}{4 \pi C}\right)^{1 / 2} \bar{m}_{W} \simeq 74 \mathrm{GeV},
$$

where $\bar{m}_{W}$ is the zero-temperature vector boson mass. Eq. (11) clearly shows that the crossover point is determined by the constant $C$, i.e., the size of the magnetic mass. The obtained value of the critical Higgs mass agrees rather well with the result of recent 
numerical simulations [7]. In contrast, for vanishing magnetic mass the first-order transition never changes to a crossover, while taking $C>1.0$ corresponding to the measured bound state mass $m_{V}$ (cf. Eq. (18), table 1) grossly underestimates $\bar{m}_{H}^{c}$.

From the effective potential (9) one can easily determine the critical line of the firstorder phase transition. For $\hat{\lambda}\left(\mu^{2}\right)<\lambda_{c}$ the conditions

$$
0=V_{1 l}\left(\sigma_{0}\right)=\left.\frac{\partial}{\partial \sigma} V_{1 l}\right|_{\sigma=\sigma_{0}}
$$

yield for the critical line $\hat{\lambda}\left(\mu^{2}\right)$,

$$
\frac{\hat{\lambda}\left(\mu^{2}\right)}{g^{2}}=\frac{1}{128 \pi^{2}} \frac{g^{4}}{\mu^{2}} .
$$

The corresponding phase diagram is shown in Fig. 1. The critical value of the mass parameter at the crossover point is given by

$$
\frac{\mu_{c}^{2}}{g^{4}} \simeq \frac{C}{8 \pi} .
$$

Using the matching relations to the finite-temperature Higgs model one can evaluate the critical temperature as function of the zero-temperature Higgs boson mass.

\section{Gauge-invariant correlation functions}

It is expected that the SU(2) Higgs model has only a single phase, and that the Higgs and the confinement regime are analytically connected [22]. All physical properties of the model can be obtained by studying correlation functions of gauge-invariant operators. This is of particular importance for numerical lattice simulations where in general the gauge is not fixed.

In the literature the following operators for scalar states with $J^{P C}=0^{++}$have been studied,

$$
\begin{aligned}
R(x) & =\operatorname{Tr}\left(\Phi^{\dagger}(x) \Phi(x)\right) \\
L(x) & =\operatorname{Tr}\left(\left(D_{\mu} \Phi\right)^{\dagger} D_{\mu} \Phi(x)\right) \\
P(x) & =\frac{1}{2} \operatorname{Tr}\left(W_{\mu \nu} W_{\mu \nu}\right)=-\frac{1}{8 g^{2}} \operatorname{Tr}\left(\left[D_{\mu}, D_{\nu}\right]\left[D_{\mu}, D_{\nu}\right]\right) .
\end{aligned}
$$

The standard operator for vector states with $J^{P C}=1^{--}$is

$$
V_{\mu}^{a}(x)=\frac{1}{2} \operatorname{Tr}\left(\Phi^{\dagger}(x) \stackrel{\leftrightarrow}{D_{\mu}} \Phi(x) \tau^{a}\right) .
$$


In the numerical simulations [9, 10, 11, 12, 13] screening masses have been determined from the 2-point functions of the operators $R$ and $V_{\mu}^{a}$,

$$
\begin{aligned}
G_{R}(x-y) & =\langle R(x) R(y)\rangle \sim e^{-m_{R}|x-y|}, \\
G_{V}(x-y)_{\mu \nu} & =\left\langle V_{\mu}(x) V_{\nu}(y)\right\rangle \sim e^{-m_{V}|x-y|} .
\end{aligned}
$$

In 111 screening masses have also been measured for the operators $L(x)$ and $P(x)$,

$$
\begin{aligned}
& G_{L}(x-y)=\langle L(x) L(y)\rangle \sim e^{-m_{L}|x-y|}, \\
& G_{P}(x-y)=\langle P(x) P(y)\rangle \sim e^{-m_{P}|x-y|} .
\end{aligned}
$$

The screening masses $m_{R}, m_{V}, m_{L}$ and $m_{P}$ have been determined for positive and negative values of $\mu^{2}$, i.e., in the symmetric phase and in the Higgs phase. In the latter case, as expected, the results agree with perturbation theory and gap equations. The value for $m_{P}$ is consistent with an intermedate state of two massive vector bosons $V$ contributing to $G_{P}$. This is the leading contribution if one expands $P(x)$ in powers of $g^{2}$.

In the symmetric phase, however, the numerical results for $m_{R}$ and $m_{V}$ do not agree with the predictions of the gap equations. Since also in the symmetric phase the vacuum expectation value of the Higgs field is different from zero, it was suggested in [6] that the magnetic mass $m_{S M}$ should determine the asymptotic behaviour of $G_{V}$ (cf. (23)). The numerical simulations show no sign of this.

What is the connection between the gauge-dependent 2-point function $G_{W}$ and the gauge-invariant 2-point function $G_{V}$ ? This question has been addressed by Fröhlich, Morchio and Strocci in their detailed study of the Higgs phenomenon in terms of gaugeinvariant operators 23]. As they have pointed out, gauge-invariant correlation functions are approximately proportional to gauge-dependent correlation functions as calculated in standard perturbation theory, if for the chosen gauge and renormalization scheme the fluctuations of the Higgs field are small compared to the vacuum expectation value. For instance, for the scalar correlation functions one has $\left(\sigma=v+\sigma^{\prime},\left\langle\sigma^{\prime}\right\rangle=0\right)$,

$$
\langle R(x) R(y)\rangle \sim v^{2}\left(\left\langle\sigma^{\prime}(x) \sigma^{\prime}(y)\right\rangle+\mathrm{O}\left(\frac{\sigma^{\prime}}{\langle\sigma\rangle}, \frac{\vec{\pi}}{\langle\sigma\rangle}\right)\right) .
$$

A measure for the relative size of the fluctuation terms is the ratio

$$
\zeta=\frac{\left\langle\Phi^{\dagger} \Phi\right\rangle}{\langle\sigma\rangle^{2}}
$$

At one-loop order one obtains in $R_{\xi}$-gauge,

$$
\begin{aligned}
\left\langle\Phi^{\dagger} \Phi\right\rangle & =\left\langle\sigma^{2}+\vec{\pi}^{2}\right\rangle=v^{2}+\left\langle\sigma^{\prime 2}+\vec{\pi}^{2}\right\rangle \\
& =v^{2}-\frac{1}{4 \pi}(M+3 \sqrt{\xi} m) .
\end{aligned}
$$




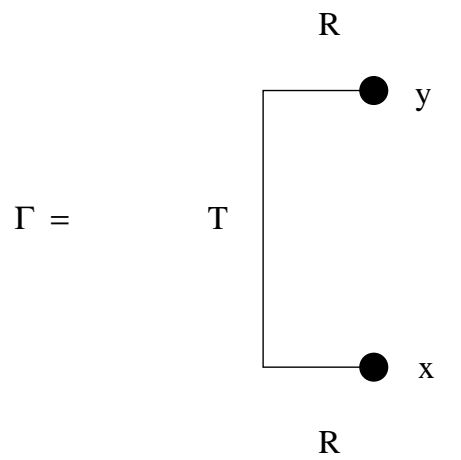

Figure 2: The path $\Gamma$ for $G_{\Phi}(T, R)$.

Here linear divergencies have been subtracted by means of dimensional regularization. Deep in the Higgs phase, where $\mu^{2}<0, v_{0}^{2}=-\mu^{2} / \lambda, M_{0}^{2}=2 \lambda v_{0}^{2}$ and $m_{0}^{2}=g^{2} v_{0}^{2} / 4$, one finds

$$
\zeta_{H}=1-\frac{3}{8 \pi}\left(\sqrt{\xi}+\frac{2}{3} \frac{\sqrt{2 \lambda}}{g}\right) \frac{g}{v}+\ldots
$$

In the relevant range of parameters one has $g / v<1$. Hence, the deviation of $\zeta_{H}$ from 1 is small and ordinary perturbation theory is reliable.

On the contrary, in the symmetric phase the situation is very different. Here the gap equations yield for the vacuum expectation value $g / v \simeq 10$. Inserting in the definition of the ratio (24) solutions of the gap equations for $M$ and $m$ one finds in the symmetric phase that $\zeta_{S M}$ deviates from 1 by more than $100 \%$. Hence, we cannot expect that the gauge-dependent 2-point functions give a good approximation to the gauge-invariant 2-point functions.

\section{Gauge-invariant screening masses}

What is the physical meaning of the propagator masses obtained from gap equations as well as numerical simulations in the symmetric phase? Several years ago the notion of a 'screening energy' has been introduced in connection with an analysis of the SU(2) Higgs model at zero temperature [24]. The authors considered the gauge-invariant correlation function

$$
G_{\Phi}(T, R)=\left\langle\operatorname{Tr}\left(\Phi^{\dagger}(y) U(\Gamma) \Phi(x)\right)\right\rangle,
$$

where

$$
U(\Gamma)=P \exp \left(i g \int_{\Gamma} d s \cdot W\right) \equiv U^{\dagger}(R, y) U(T) U(R, x),
$$

and the path $\Gamma$

$$
\Gamma \equiv \Gamma(y, R) \circ \Gamma(T) \circ \Gamma(R, x)
$$

is shown in Fig. 2. For large $T$, with $R$ fixed, an exponential fall-off was found,

$$
G_{\Phi}(T, R) \sim e^{-m_{\Phi} T},
$$


with $m_{\Phi}$ being independent of $R$. In temporal gauge the 2-point function takes the form,

$$
G_{\Phi}(T, R)=\left\langle\operatorname{Tr}\left(\Phi^{\dagger}(x) U^{\dagger}(R, x) e^{-H T} U(R, x) \Phi(x)\right)\right\rangle
$$

where $H$ is the hamiltonian. Comparison of Eqs. (31) and (32) suggests that $m_{\Phi}$ is the energy of a dynamical charge bound by an external charge [24]. If the energy of the infinitely heavy external charge is properly subtracted, $m_{\Phi}$ corresponds to the 'constituent', or screening mass of the bound scalar $\Phi$. In the case $R=0$ the 2-point function $G_{\Phi}$ reduces to the gauge-invariant propagator

$$
\hat{G}_{\Phi}(x-y)=\left\langle\operatorname{Tr}\left(\Phi^{\dagger}(y) U_{y x} \Phi(x)\right)\right\rangle \sim e^{-m_{\Phi}|x-y|},
$$

where $U_{y x}$ is the non-abelian phase factor along the straight line from $x$ to $y$.

The definition of a screening mass for the vector boson is completely analogous. The obvious definiton is

$$
\hat{G}_{W}(x-y)_{\mu \nu \rho \sigma}=\left\langle W_{\mu \nu}^{T}(y) U_{y x}^{\mathcal{A}} W_{\rho \sigma}(x)\right\rangle \sim e^{-m_{W}|x-y|} .
$$

where the superscript $\mathcal{A}$ denotes $\mathrm{SU}(2)$ matrices in the adjoint representation.

The contribution from the phase factor to the masses $m_{\Phi}$ and $m_{W}$, which depend on the mass parameter $\mu^{2}$, are linearly divergent. Renormalized screening masses can be defined by matching $m_{\Phi}$ and $m_{W}$ to the masses $m_{R}$ and $m_{V}$ of the gauge-invariant correlation function at some value $\mu_{0}^{2}$ in the Higgs phase. The corresponding screening masses $m_{\Phi}\left(\mu^{2} ; \mu_{0}^{2}\right)$ and $\mu_{W}^{2}\left(\mu^{2} ; \mu_{0}^{2}\right)$ satisfy the boundary conditions

$$
m_{\Phi}\left(\mu_{0}^{2} ; \mu_{0}^{2}\right)=m_{R}\left(\mu_{0}^{2}\right) \quad, \quad m_{W}\left(\mu_{0}^{2} ; \mu_{0}^{2}\right)=m_{V}\left(\mu_{0}^{2}\right) .
$$

These screening masses, as functions of $\mu^{2}$, should essentially coincide with the solutions $M\left(\mu^{2}\right)$ and $m\left(\mu^{2}\right)$ of the gap equations, respectively.

What is the role of the screening masses in the correlation functions of gauge-invariant operators? As discussed above, the fluctuations dominate in the symmetric phase. Hence, one may expect that multi-particle states of 'constituent' scalar and vector bosons dominate the exponential fall-off of the 2-point functions. This is in the spirit of previously proposed bound state models [25, 26]. According to Fig. 3, for $G_{R}$ this should be a $\left(\Phi^{\dagger} \Phi\right)$ state (a), for $G_{L}$ a $\left(\Phi^{\dagger} W W \Phi\right)$ state (b), for $G_{P}$ a $(W W W W)$ state (c) and for $G_{V}$ a $\left(\Phi^{\dagger} W \Phi\right)$ state $(\mathrm{d})$. Here we have identified a covariant derivative $D_{\mu}$ with a constituent vector boson $W$, since for bound states in the symmetric phase an expansion in powers of $g^{2}$ is not justified. Neglecting binding effects, this yields the mass formulae

$$
m_{R} \simeq 2 m_{\Phi} \quad m_{L} \simeq 2 m_{\Phi}+2 m_{W} \quad m_{P} \simeq 4 m_{W} \quad m_{V} \simeq 2 m_{\Phi}+m_{W} .
$$




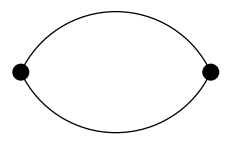

a)

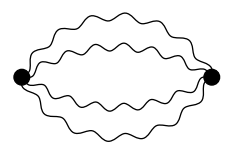

c)

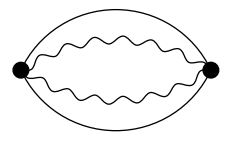

b)

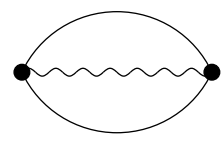

d)

Figure 3: Two-point functions to leading order for a constituent model.

These relations can be compared with results from lattice simulations. A screening mass $m_{W}$ for the vector boson was determined in [12], $m_{W}=0.35(1) g^{2}$. No scalar screening mass has been measured so far, hence we choose $m_{\Phi}=m_{R} / 2$. This yields three predictions for $m_{L}, m_{P}$ and $m_{V}$ which are compared with the results of [1] in table 1 . The qualitative agreement supports the constituent picture.

\begin{tabular}{|c|ccc|c|}
\hline & \multicolumn{3}{|c|}{$J^{P C}=0^{++}$} & $J^{P C}=1^{--}$ \\
& $R$ & $L$ & $P$ & $V$ \\
\hline \hline lattice simulations [1] & $0.839(15)$ & $1.47(4)$ & $1.60(4)$ & $1.27(6)$ \\
\hline constituent model & - & 1.54 & 1.40 & 1.18 \\
\hline
\end{tabular}

Table 1: Comparison of screening masses from lattice simulations and a constituent model. $m_{R}$ is used to fix the constituent scalar mass.

The proposed picture can be tested by measuring the gauge-invariant propagators $\hat{G}_{\Phi}$ and $\hat{G}_{W}$ as functions of $\mu^{2}$. The masses $m_{\Phi}\left(\mu^{2} ; \mu_{0}^{2}\right)$ and $m_{W}\left(\mu^{2} ; \mu_{0}^{2}\right)$ should behave like the solutions $M\left(\mu^{2}\right)$ and $m\left(\mu^{2}\right)$ of the gap equations. In particular, at a first-order transition from the Higgs phase to the symmetric phase, both screening masses should jump to smaller values. With increasing $\lambda / g^{2}$ the jump should decrease and eventually 
vanish at the critical coupling where the crossover behaviour sets in.

\section{Acknowledgements}

We would like to thank K. Jansen, M. Laine, M. Lüscher, I. Montvay and M. Teper for helpful discussions.

\section{References}

[1] D. A. Kirzhnits, JETP Lett. 15 (1972) 529;

D. A. Kirzhnits and A. D. Linde, Phys. Lett. B42 (1972) 471

[2] V. A. Kuzmin, V. A. Rubakov and M. E. Shaposhnikov, Phys. Lett. B155 (1985) 36

[3] K. Jansen, Nucl. Phys. B(Proc. Suppl.) 47 (1996) 196;

K. Rummukainen, hep-lat/9608079;

W. Buchmüller, DESY 96-216, hep-ph/9610335

[4] A. D. Linde, Phys. Lett. B96 (1980) 289;

D. Gross, R. Pisarski and L. Yaffe, Rev. Mod. Phys. 53 (1981) 43

[5] A. Jakovác, K. Kajantie and A. Patkós, Phys. Rev. D49 (1994) 6810;

K. Farakos, K. Kajantie, K. Rummukainen and M. Shaposhnikov, Nucl. Phys. B425 (1994) 67

[6] W. Buchmüller and O. Philipsen, Nucl. Phys. B443 (1995) 47

[7] K. Kajantie, M. Laine, K. Rummukainen and M. Shaposhnikov, Phys. Rev. Lett. 77 (1996) 2887

[8] F. Karsch, T. Neuhaus, A. Patkós and J. Rank, FSU-SCRI-96C-79, hep-lat/9608087

[9] Z. Fodor, J. Hein, K. Jansen, A. Jaster and I. Montvay, Nucl. Phys. B439 (1995) 147

[10] K. Kajantie, M. Laine, K. Rummukainen and M. Shaposhnikov, Nucl. Phys. B466 (1996) 189

[11] O. Philipsen, M. Teper and H. Wittig, Nucl. Phys. B469 (1996) 445 
[12] F. Karsch, T. Neuhaus, A. Patkós and J. Rank, Nucl. Phys. B474 (1996) 217

[13] M. Gürtler, H. Perlt, A. Schiller, E.-M. Ilgenfritz and J. Kripfganz, DESY 96-113, hep-lat/9605042

[14] A. K. Rebhan, Phys. Rev. D48 (1993) 3967

[15] W. Buchmüller, Z. Fodor, T. Helbig and D. Walliser, Ann. Phys. 234 (1994) 260;

J. R. Espinosa, M. Quirós and F. Zwirner, Phys. Lett. B314 (1993) 206

[16] G. Alexanian and V. P. Nair, Phys. Lett. B352 (1995) 435

[17] R. Jackiw and S.-Y. Pi, Phys. Lett. B368 (1996) 131

[18] M. Reuter and C. Wetterich, Nucl. Phys. B408 (1993) 91

[19] W. Buchmüller and O. Philipsen, Phys. Lett. B354 (1995) 403

[20] H. G. Evertz, J. Jersák, K. Kanaya, Nucl. Phys. B285[FS19] (1987) 229

[21] N. Tetradis, CERN-TH/96-190, hep-ph/9608272;

B. Bergerhoff and C. Wetterich, HD-THEP-96-51, hep-ph/9611462

[22] K. Osterwalder and E. Seiler, Ann. Phys. 110 (1978) 440;

E. Fradkin and S. H. Shenker, Phys. Rev. D19 (1979) 3682

[23] J. Fröhlich, G. Morchio and F. Strocchi, Nucl. Phys. B190[FS3](1981) 553

[24] H. G. Evertz, V. Grösch, J. Jersák, H. A. Kastrup, T. Neuhaus, D. P. Landau and J.-L. Xu, Phys. Lett. B175 (1986) 335

[25] B. Grossmann, S. Gupta, U. M. Heller and F. Karsch, Nucl. Phys. B417 (1994) 289

[26] H.-G. Dosch, J. Kripfganz, A. Laser and M. G. Schmidt, Phys. Lett. B365 (1995) 213 